\title{
Estilo de vida saludable, nivel de glucosa e IMC en adultos del Valle de Amauta - Ate Vitarte - Lima - 2020
}

\author{
Healthy lifestyle, glucose level and BMI in adults from the Amauta Valley - Ate \\ Vitarte - Lima - 2020
}

\section{Carla Hurtado Casanca ${ }^{1}$, Noelene Atoc Ventocilla², Mery Rodríguez Vásquez ${ }^{3}$}

\section{RESUMEN}

Objetivo: Determinar la relación entre los estilos de vida saludable, glucosa e IMC en adultos del Valle de Amauta - Ate Vitarte - Lima - 2020. Metodología: Diseño no experimental, enfoque cuantitativo, de corte transversal y de tipo correlacional. La muestra está compuesta por a 175 adultos de ambos sexos de edades entre 18 a 64 años. Se recolectaron los datos a través de un cuestionario sobre estilo de vida; además, se tomaron medidas antropométricas como peso y talla para determinar el Índice de masa corporal (IMC), así mismo, se evaluó el nivel de glucosa. Resultados: El 82.7\% de la población evaluada presentó un estilo de vida saludables y solo el $17.7 \%$ una condición poco saludable; así mismo, el $77.2 \%$ no realizaba actividad física, encontrando que el $52.6 \%$ realiza actividad ligera. En cuanto al consumo de alcohol y otras drogas, el $94.2 \%$ es saludable; también, el $76.0 \%$ informó tener un sueño adecuado y el $24.0 \%$ tenía una mala calidad de sueño. En cuanto a los hábitos alimentarios, $81.2 \%$ es saludable, un grupo de $18.9 \%$ no tiene hábitos saludables, también el $68.0 \%$ no tenía un autocuidado. Se encontró que el $42.3 \%$ presentó diabetes. Finalmente, el $34.3 \%$ tenía un IMC normal, $42.3 \%$ presentó sobrepeso y $23.4 \%$ presentó obesidad. Conclusión: Existe relación entre el estilo de vida, los niveles de glucosa y el IMC de los participantes. Además, el estudio demuestra que la falta de actividad física está relacionado al aumento del IMC, que a la vez se asocia a los niveles de glucosa elevados. Por lo tanto, si se hace una modificación del estilo de vida, condición física y deporte, recreación y manejo del tiempo libre, consumo de alcohol y otras drogas, sueño, hábitos alimentarios y autocuidado, y cuidado médico, puede ser de gran ayuda para la prevención de enfermedades metabólicas.

Palabras clave: Estilo de vida, IMC, glucosa.

\section{ABSTRACT}

Objective: To determine the relationship between healthy lifestyles, glucose and BMI in adults from the Amauta Valley - Ate Vitarte - Lima - 2020. Methodology: Non-experimental design, quantitative, crosssectional and correlational approach. The sample is made up of 175 adults of both sexes between the ages of 18 and 64 years. Data were collected through a lifestyle questionnaire; Furthermore, anthropometric measurements such as weight and height were taken to determine the Body Mass Index (BMI), and the glucose level was also evaluated. Results: $82.7 \%$ of the evaluated population presented a healthy lifestyle and only $17.7 \%$ an unhealthy condition; likewise, $77.2 \%$ did not perform physical activity, finding that $52.6 \%$ performed light activity. Regarding the consumption of alcohol and other drugs, $94.2 \%$ are healthy; Also, $76.0 \%$ reported having adequate sleep and $24.0 \%$ had poor

${ }^{1}$ Policlinico DafiSalud: Lima, Perú.

Orcid ID: 0000-0002-0995-2062

${ }^{2}$ Universidad Peruana Unión, Lima, Perú

Orcid ID: 0000-0001-8754-5481

3Universidad Peruana Unión, Lima, Perú

Orcid ID: 0000000326167979 
sleep quality. Regarding eating habits, $81.2 \%$ are healthy, a group of $18.9 \%$ does not have healthy habits, and $68.0 \%$ did not have self-care. It was found that $42.3 \%$ had diabetes. Finally, $34.3 \%$ had a normal BMI, $42.3 \%$ were overweight, and $23.4 \%$ were obese. Conclusion: There is a relationship between the lifestyle, glucose levels and BMI of the participants. In addition, the study shows that lack of physical activity is related to increased BMI, which in turn is associated with elevated glucose levels. Therefore, if a modification of the lifestyle, physical condition and sports, recreation and free time management, consumption of alcohol and other drugs, sleep, eating habits and self-care, and medical care is made, it can be of great help to the prevention of metabolic diseases.

Keywords: Lifestyle, BMI, glucose.

\section{INTRODUCCIÓN}

Las enfermedades crónicas no transmisibles (ENT) son afecciones de larga duración, las cuales se deben a diversos factores como genéticos, fisiológicos, ambientales y conductuales. Las causas de las ENT son multifactoriales, entre las más destacadas se encuentran una vida poco saludable, dietas inadecuadas, la falta de actividad física, el consumo de tabaco y el uso nocivo de alcohol.

Los adultos, con niveles elevados de glucosa y un IMC fuera de los rangos establecidos, corren mayor riesgo al presentar ENT tales como hipertensión, obesidad, diabetes mellitus tipo 2 , enfermedades cardiovasculares y diferentes tipos de cáncer. La Organización Mundial de la Salud (OMS) (2018), declaró "que las ENT cobran la vida de 41 millones de personas cada año, lo que equivale a un $71 \%$ de las muertes que se dan en el mundo. Así mismo, cada año mueren 15 millones de personas de las edades de 30 a 69 años, en los países de ingresos bajos y medio". La OMS (2020) declaró "que 2.8 millones de personas mueren cada año por causa de obesidad y sobrepeso; también, el $39 \%$ las personas de 18 a más edad tienen sobrepeso y el $13 \%$ son obesos". La Federación Internacional de Diabetes informa (2019), afirma que "425 millones de adultos tenían diabetes". En América Latina, de acuerdo a la Organización Panamericana de la Salud (OPS) (2018) "cerca de $58 \%$ de los habitantes vive con sobrepeso; así mismo, la obesidad afecta a 140 millones de personas, esto es un $23 \%$ de la población".

Por otro lado, en Perú, según el Instituto Nacional de la Salud (INS), "para el año 2019, se estima que el $69.9 \%$ de adultos tenían sobrepeso y obesidad, afectando a los escolares, adolescentes, adultos jóvenes y adultos mayores en un $32.3 \%, 23.9 \%, 42.4 \%$ y $33.1 \%$, respectivamente". El Ministerio de Salud, en el periodo 2005 a 2018, informó que la prevalencia nacional de diabetes mellitus tipo 2 se incrementó de $2.1 \%$ a $22.1 \%$ casos por cada 100000 habitantes; el sexo femenino es el de mayor prevalencia. Asimismo, las regiones geográficas con mayor prevalencia fueron la Costa de $3.8 \%$ a $35.3 \%$ por cada 100.000 habitantes y Selva de $1.1 \%$ a $22.1 \%$ por cada 100.000 habitantes. Los departamentos con mayor incremento en la prevalencia fueron Cajamarca (1733.3\%) y Puno (1704.2\%), observándose departamentos como Tumbes, Ica, Lambayeque, Callao, Lima, Loreto y Madre de Dios con prevalencias mayores al promedio nacional (Ccorahua Et al,2019).

La obesidad es una enfermedad compleja que consiste en tener una cantidad excesiva de grasa corporal; se caracteriza por un índice de masa corporal igual o superior a treinta. Las causas pueden ser por genética, conductuales, metabólicas y hormonales. También al ingerir más calorías, teniendo menos desgaste, como la actividad física; así mismo, la ingesta de alimentos ricos en grasa, sal y azúcares, siendo pobres en vitaminas, minerales y otros micronutrientes, otro factor es el sedentarismo y eso se debe a los métodos modernos, trabajo en oficinas, transporte y etc. La consecuencia de tener obesidad aumenta a tener problemas médicos aumenta el riesgo de enfermedades y problemas de salud, tales como enfermedad cardíaca, diabetes, presión arterial alta y ciertos tipos de cáncer. Si la persona sufre de obesidad tiene mayor riesgo de sufrir diabetes (Moreno, 2012).

Prediabetes son niveles de glucemia, que se encuentra por encima de los valores normales, pero por debajo de los niveles para clasificar como diabetes, se considera riesgo para la predicción de diabetes y complicaciones vasculares (Diaz et al, 2011). 
La diabetes es una enfermedad crónica que aparece cuando el páncreas no produce insulina suficiente o cuando el organismo no utiliza eficazmente la insulina que produce. El efecto de la diabetes no controlada es la hiperglucemia (aumento del azúcar en la sangre). La causa se sufrir diabetes es tener sobrepeso u obesidad. Según un estudio realizado en Estados Unidos, por cada kilo de peso que gana la gente, aumenta en $4.5 \%$ la probabilidad de desarrollar diabetes (Durán et al, 2012). Otra causa es genética, ya que las personas con antecedentes familiares son más propensos a sufrir con diabetes, vida sedentaria, esto conlleva la falta de ejercicio o actividad física contribuyendo a acumular mayor grasa abdominal; además, no realizar actividad física genera que se ralentice el metabolismo de la glucosa y los hábitos alimentarios inadecuados como una dieta pobre e inadecuada, siendo el consumo de alimentos ricos en hidratos de carbono y grasa provoca alteraciones en los niveles de glucosa y en la producción de insulina. Las consecuencias de la diabetes no tienen curación, pero sí se puede controlar; afecta a la visión, a los riñones, provoca infarto de miocardio o trombosis y hemorragia cerebral (SEMI, 2019).

Es evidente que los malos hábitos alimentarios de esta tesis pretenden exponer que los hábitos alimentarios, tiene que ver mucho con el trastorno desobrepesoyobesidad, causandolaenfermedad de la diabetes. En la revisión analizaremos estudios desarrollados, enfocándonos a mejorar los hábitos de alimentación para lograr tratar y prevenir su aparición de dicha enfermedad. Este trabajo tiene como objetivo determinar la relación entre los estilos de vida saludable, nivel de glucosa en IMC en adultos.

\section{METODOLOGÍA}

La presente investigación corresponde a un diseño no experimental y de corte transversal, debido a que no se han manipulado variables y los datos serán tomados en un solo momento. El alcance del estudio es correlacional, ya que se busca identificar si existe relación entre la identidad de género y el funcionamiento familiar (Hernández, Fernández y Baptista, 2014).

\section{Participantes}

La muestrafue no probabilística porconveniencia, estuvo conformada por 175 adultos de ambos sexos de edades entre 18 a 64 años.

\section{Instrumentos}

Se utilizó para la recolección de datos: cuestionario sobre estilos de vida saludable, ficha de registro antropométrico (peso, talla, $I M C$, registro de nivel de glucosa y el registro de datos demográficos.

\section{Ficha de registro}

En los datos demográficos se tomó en cuenta la edad de la persona (mayores de 18 hasta los 64 años), el sexo si es femenino o masculino, estado civil, lugar de procedencia, condición laboral, ocupación, actividad que ejercen y antecedentes patológicos (para nuestro estudio se seleccionó sin antecedente patológico).

\section{Cuestionario sobre estilos de vida saludable}

El cuestionario empleado comprende seis secciones. Se evaluó las condiciones de actividad física, la recreación y manejo del tiempo libre, el consumo de alcohol, tabaco y otras drogas, el sueño, los hábitos alimenticios y autocuidado, y por último el cuidado médico. Se realizaron las preguntas según los ítems, con las opciones para marcar como: siempre, frecuentemente, algunas veces y nunca. Cada cuestionario fue entregado a la persona $y$ contestada de manera personal.

\section{Ficha de registro antropométrico}

La recolección de los datos antropométricos se realizó siguiendo las normas de la Guía Técnica para la Valoración Nutricional Antropométrica de la Persona Adulta. Para la evaluación del peso, se utilizó la balanza de tipo reloj calibrado, ubicada en una superficie plana, lisa y horizontal. Se solicitó, a la persona, quitarse los zapatos y el exceso de ropa. Para la medición de talla, se utilizó el tallímetro fijo de madera, se colocó en una superficie lisa y plana; de igual manera, se verificó el tope móvil para que deslice suavemente, se procedió a la medición de talla, explicando a la persona el procedimiento y solicitando su colaboración; así mismo, se le pidió que se quite los zapatos, exceso de ropa, accesorios u otros objetos en la cabeza o cuerpo que interfieran con la medición; se le indicó dónde tenía que estar en el tablero: de espaldas en el tablero con una posición erguida, de mirada al frente, con brazos al costado del cuerpo con 
las palmas de las manos descansando sobre los muslos, los talones juntos y las puntas de los pies ligeramente separados; luego, se procedió a medir con el tope móvil del tallímetro hasta hacer contacto con la superficie superior de la cabeza. Se evaluó el IMC según el índice de Quetelet y la clasificación fue según la Guía Técnica para la Valoración Nutricional Antropométrica de la Persona Adulta del Ministerio de Salud del Perú (MINSA): bajo peso, $\leq 18.5$; normopeso, $\geq 18.5$ $\leq 24.9 \mathrm{~kg} / \mathrm{m} 2$; sobrepeso, entre 25.0 a $29.9 \mathrm{~kg} /$ $\mathrm{m} 2$; obesidad grado I, entre $\geq 30-\leq 34.9 \mathrm{~kg} /$ $\mathrm{m} 2$; obesidad grado II, entre $\geq 35-\leq 39.9 \mathrm{~kg} / \mathrm{m} 2$ y Obesidad III, $\geq 40$ kg/m2.

\section{Muestra de glucosa:}

Se utilizó un glucómetro digital con sus respectivas tiras reactivas y lancetas. Se le solicitó a la persona que debía estar en ayunas; luego, se procedió a desinfectar un dedo con ayuda de un algodón y alcohol; una vez lista, se procedió a pinchar con la lanceta y tomar la muestra con la tira y colocar en el glucómetro, se esperó el resultado unos segundos. Fue clasificado de la siguiente manera: $<99 \mathrm{mg} / \mathrm{dL}$ niveles bajos, $100-125 \mathrm{mg} / \mathrm{dL}$ nivel normal y $>126 \mathrm{mg} / \mathrm{dL}$ elevado

\section{Análisis de datos}

Para el registro y orden de datos, se ingresaron los datos en el programa de Microsoft Excel versión 2013. Para el procesamiento y análisis de datos, se utilizó el programa SPSS versión 25. Para ver la distribución y relación de variables, se utilizó la prueba estadística de Rho de Spearman.

\section{RESULTADOS}

Para una mejor comprensión del perfil sociodemográfico, se realizaron tablas descriptivas, con la finalidad de encontrar posibles relaciones en los datos recolectados.

De acuerdo a la tabla 1, la muestra estuvo formada por 175 personas de ambos géneros, $71.4 \%$ correspondiente al género femenino y el $28.6 \%$ al género masculino, el $74.9 \%$ tienen entre 30 a 59 años y el $25.1 \%$ de 18 a 29 años. El $63.4 \%$ era soltera y solo un $36.6 \%$ estaban casados. Un gran número de participantes, $58.3 \%$ es de la sierra, $30.3 \%$ de la costa, el $10.3 \%$ de la selva y un $1.1 \%$ del extranjero. Con respecto a la condición laboral, la mayoría de las personas, $52 \%$ son de auto sostén y el $48 \%$ dependientes. Asimismo, el 29.1\% tiene de ocupación comerciante, $26.3 \%$ de otros, $22.9 \%$ de obrero y el $21.7 \%$ de ama de casa.

Tabla 1

Características sociodemográficas de la muestra

\begin{tabular}{lll} 
Variable & $\mathrm{n}$ & $\%$ \\
\hline Edad & 44 & 25.1 \\
18 a 29 años & 131 & 74.9 \\
30 a 59 años & & \\
Sexo & 50 & 28.6 \\
Masculino & 125 & 71.4 \\
Femenino & & 63.4 \\
Estado civil & 111 & 36.6 \\
Soltero & 64 & \\
Casado & & 30.3 \\
Lugar de procedencia & 53 & 58.3 \\
Costa & 102 &
\end{tabular}




\begin{tabular}{lll} 
Selva & 18 & 10.3 \\
Extranjero & 2 & 1.1 \\
Condición laboral & & \\
Dependiente & 84 & 48.0 \\
Autosostén & 91 & 52.0 \\
Ocupación & & \\
Ama de casa & 38 & 21.7 \\
Comerciante & 51 & 29.1 \\
Obrero & 40 & 22.9 \\
Otros & 46 & 26.3 \\
\hline
\end{tabular}

La tabla 2 muestra, en cuanto al estilo de vida, un $78.9 \%$ en estado saludable, $17.7 \%$ poco saludable y un $3.4 \%$ muy saludable. Con relación a la actividad física, el $40.6 \%$ de la población era poco saludable, $36.6 \%$ no saludable, $20 \%$ saludable y el $2.9 \%$ muy saludable.En cuanto a recreación, se obtuvo que el $42.3 \%$ es poco saludable, el $44.6 \%$ saludable, también, el $8 \%$ muy saludable y solo el $5.1 \%$ no saludable. Analizando los resultados con respecto al consumo de alcohol se obtuvo que, el $65,1 \%$ muy saludable, $29.1 \%$ saludable, $5.7 \%$ poco saludable.
En los resultados de la variable de sueño, se encontró que el $58.9 \%$ y $17.1 \%$ son saludables y muy saludables, encontrándose también que $23,4 \%$ poco saludable y el $6 \%$ no saludable. En otro apartado, fueron preguntados por sus hábitos alimentarios, se detectó que $74.9 \%$ y $18.9 \%$ son saludables y poco saludables y $6.3 \%$ muy saludables.

En la tabla, también se encuentra el autocuidado poco saludable $49.1 \%$ y 18.9 no saludable; así mismo, $25.1 \%$ saludable y $6.9 \%$ muy saludable.

Tabla 2

Estilos de vida saludable

$\begin{array}{lll}\text { Variable } & n & \% \\ \text { Estilos de vida } & & 0 \\ \text { No saludable } & 0 & 17.7 \\ \text { Poco saludable } & 31 & 78.9 \\ \text { Saludable } & 138 & 3.4 \\ \text { Muy saludable } & 6 & \\ \text { Condición actividad física y deporte } & & 36.6 \\ \text { No saludable } & 64 & 40.6 \\ \text { Poco saludable } & 71 & 20.0 \\ \text { Saludable } & 35 & 2.9 \\ \text { Muy saludable } & 5 & \\ \text { Recreación y manejo del tiempo libre } & & 5.1 \\ \text { No saludable } & 9 & 42.3 \\ \text { Poco saludable } & 74 & 44.6 \\ \text { Saludable } & 78 & 8.0 \\ \text { Muy saludable } & 14 & \end{array}$




\begin{tabular}{lll} 
Consumo de alcohol, tabaco y otras drogas & & 0 \\
No saludable & 0 & 5.7 \\
Poco saludable & 10 & 29.1 \\
Saludable & 51 & 65.1 \\
Muy saludable & 114 & \\
Sueño & &, 6 \\
No saludable & 1 & 23.4 \\
Poco saludable & 41 & 58.9 \\
Saludable & 103 & 17.1 \\
Muy saludable & 30 & \\
Hábitos alimentarios & & 0 \\
No saludable & 0 & 18.9 \\
Poco saludable & 33 & 74.9 \\
Saludable & 131 & 6.3 \\
Muy saludable & 11 & \\
Autocuidado y cuidado médico & & 18.9 \\
No saludable & 33 & 49.1 \\
Poco saludable & 86 & 25.1 \\
Saludable & 44 & 6.9 \\
Muy saludable & 12 & 100.0 \\
Total & 175 & \\
\hline
\end{tabular}

En la tabla 3 , se observa que el $27.4 \%$ y $14.9 \%$ presenta prediabetes y diabetes; también, un $57.7 \%$ tiene como resultado normal.

Tabla 3

Glucosa

\begin{tabular}{lll} 
Glucosa & $\mathrm{n}$ & $\%$ \\
Normal & 101 & 57.7 \\
Pre-diabetes & 48 & 27.4 \\
Diabetes & 26 & 14.9 \\
Total & 175 & 100.0 \\
\hline
\end{tabular}

En la tabla 4, se evaluó el índice de masa corporal y fueron clasificados como el $42,3 \%$ con sobrepeso; en cuanto a obesidad grado I, II y III con un $17.1 \%, 5.7 \%$ y $0.6 \%$, se encontró también con un $34.3 \%$ en estado normal. 
Tabla 4

Índice de Masa Corporal

\begin{tabular}{lll} 
IMC & $\mathrm{n}$ & $\%$ \\
\hline Normal & 60 & 34.3 \\
Sobrepeso & 74 & 42.3 \\
Obesidad Grado I & 30 & 17.1 \\
Obesidad Grado II & 10 & 5.7 \\
Obesidad Grado III & 1 & 0.6 \\
Total & 175 & 100.0 \\
\hline
\end{tabular}

En la tabla 5, se registra el análisis correlacional de las variables de estudio. Se encontró correlación estadísticamente significativa entre el estilo de vida y el IMC de los participantes ( $r h o=-0.331 ; p=0.000$ ). También, se evidenció que el estilo de vida está correlacionado con el nivel de glucosa (rho=-0.232, $\mathrm{p}=0.002$ ). Se observa que la Condición física y deporte tienen correlación significativa con el IMC y el nivel de glucosa ( $r h o=-0.249 ; p=0.001)$ y $(r h o=-0.271 ; p=0.000)$; así mismo, la recreación y manejo del tiempo libre tienen correlación significativa con el nivel de glucosa y el IMC (rho=-0,166, $p=0,0028)$, (rho=-0.322; $p=0.000$ ) no se encontró correlación entre el consumo de alcohol, tabaco y otras drogas con el IMC y el nivel de glucosa (rho=-0.040; $p=0.603$ y rho=-0.028, $p=0,709$ ). Se evidencia que el sueño está correlacionado significativa con el nivel de glucosa y IMC (rho $=-0,174, p=0.021)$, al mismo tiempo, los hábitos alimenticios están relacionados estadísticamente entre IMC ( $\mathrm{rho}=-0,170$, $p=0,025)$, pero no hay correlación entre hábitos alimentarios con el nivel de glucosa ( $r h o=-0,076$, $p=0,316$ ) y el autocuidado y cuidado médico se encontró que hay correlación en el nivel de glucosa y IMC (rho=-0.207; $p=0.006)$ y $($ rho $=-0,152, p=0,044)$.

Tabla 5

Correlación entre el estilo de vida, glucosa e IMC

\begin{tabular}{|c|c|c|c|c|}
\hline & & & Glucosa & IMC \\
\hline \multirow{15}{*}{ Rho de Spearman } & Estilos de vida & Coeficiente de correlación &,- 232 &,- 331 \\
\hline & & $\mathrm{p}$ & 0.002 & 0.000 \\
\hline & & $\mathrm{n}$ & 175 & 175 \\
\hline & $\begin{array}{l}\text { Condición física y } \\
\text { deporte }\end{array}$ & Coeficiente de correlación &,- 271 &,- 249 \\
\hline & & $\mathrm{p}$ & 0.000 & 0.001 \\
\hline & & $\mathrm{n}$ & 175 & 175 \\
\hline & $\begin{array}{l}\text { Recreación y manejo } \\
\text { de tiempo libre }\end{array}$ & Coeficiente de correlación &,- 166 &,- 322 \\
\hline & & $\mathrm{p}$ & 0.028 & 0.000 \\
\hline & & $\mathrm{n}$ & 175 & 175 \\
\hline & $\begin{array}{l}\text { Consumo de alco- } \\
\text { hol, tabaco y otras } \\
\text { drogas }\end{array}$ & Coeficiente de correlación & -0.040 & -0.028 \\
\hline & & $\mathrm{p}$ & 0.603 & 0.709 \\
\hline & & $\mathrm{n}$ & 175 & 175 \\
\hline & Sueño & Coeficiente de correlación &,- 156 &,- 174 \\
\hline & & $\mathrm{p}$ & 0.039 & 0.021 \\
\hline & & $\mathrm{n}$ & 175 & 175 \\
\hline
\end{tabular}




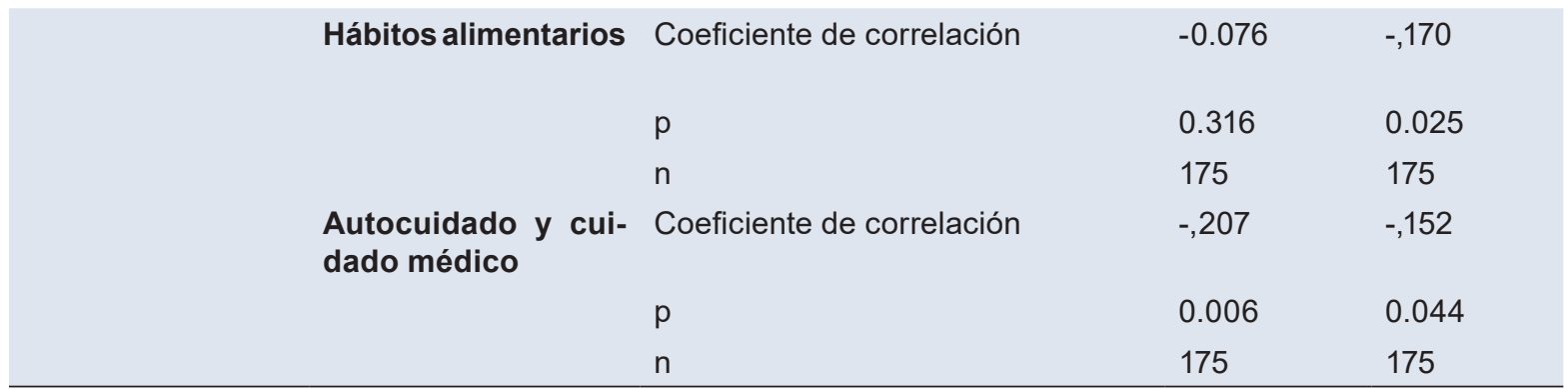

\section{DISCUSIÓN}

En este estudio, se evidenció relación entre las variables de estilos de vida saludable, glucosa e IMC en una población adulta. Se observa en la tabla 1, los resultados sociodemográficos, encontrando que la mayor proporción de la muestra fue las mujeres. Resultados similares fueron reportados por Reynaga et al (2016), "en los que se observaron que la población estudiada en su mayoría eran mujeres. También los participantes eran de edades de 30 a 59 años la mayoría eran solteros, procedentes de la sierra, con condición laboral de autosostén y en su mayoría comerciantes". Así también, lo demuestra Elizondo et al. (2005), "que los factores sociodemográficos como sexo, edad, estudios, profesión y estado civil parecen ser determinantes del estilo de vida",

Este estudio demuestra que el $78.9 \%$ de la población tenía un estilo de vida "saludable", mientras que solo el $3.4 \%$ es "muy saludable". Resultados casi similares a la presente investigación fueron presentados por Aguirre et al. (2015), en los que se evidenciaron que el $83 \%$ de la muestra presentó un estilo de vida adecuado, casi similar, a los estudios de Triviño et al. (2009), muestran que, solo el $56.5 \%$ tiene un buen estilo de vida. Se evidenció también, en este estudio, que ningún participante presenta un estilo de vida "no saludable" y solo el $17.7 \%$ "poco saludable", datos congruentes con los presentados por Silva et al.(2017), refiriendo un estilo de vida no saludable para un grupo minoritario, lo cual Ribeiro et al.(2019), con respecto al estilo de vida, en su estudio evidencia una alta prevalencia de malos hábitos alimenticios, en discordancia a lo reportado, Barragán et al.(2015), muestra que el $66.40 \%$ presenta un estilo de vida regular. En la revisión de Mora (2012), menciona que la práctica de estilos de vida, ayuda a mejorar la calidad de vida para reducir el riesgo de desarrollar enfermedades crónicas.
Además, se encontró una correlación entre el estilo de vida, IMC y glucosa como se observa en la tabla 5. Estos resultados son casi similares los reportados por Reséndiz et al. (2010), quienes en su investigación encontraron valores semejantes al estudio, encontrando una prevalencia de sobrepeso tanto en mujeres y varones, esta población no practica ningún deporte o ejercicio, entonces es indudable que el estilo de vida tiene un impacto en la salud de los adultos. Por otro lado, en la investigación de Ramírez et al. (2011) demuestran la influencia del nivel de glucosa en el estilo de vida en pacientes con diabetes mellitus II, con la aplicación de un cuestionario de estilo de vida, mostrando que el $80 \%$ practica un estilo de vida saludable, lo cual ayuda a prevenir sus complicaciones en relación a su enfermedad. Entonces se infiere que el estilo de vida es un conjunto de decisiones que puede afectar o beneficiar la salud, con algún grado de control voluntario.

Referente a la actividad física y deporte, se muestra que el $22.9 \%$ de la población es "saludable y muy saludable", valores casi similares a los informados por Reynaga et al. (2016), en los que el $13.5 \%$ sí practica ejercicio o deporte. Por otra parte, la investigación evidencia que el $77.2 \%$ presenta una actividad física y deporte "no saludable y poco saludable"; de la misma forma, Reynaga et al. (2016). en su investigación. mostraron valores elevados en la población evaluada, donde el $86.5 \%$ no realizaban actividad física. Estos valores, se pueden relacionar con las largas horas de trabajo y poca actividad física a la que está sometida la población, así lo demuestra el estudio realizado por Álvarez et al. (2016), donde más del $54 \%$ permanece sentado entre 7 y 12 horas diariamente, indicando un menor tiempo para realizar ejercicio. De la misma forma, Molina et al. (2016), en su población estudiada, manifestaron pasar sentados, entre 5 a 8 horas al día, teniendo una mayor conducta sedentaria. 
Como también en estudios de Velandia et al. (2015) mencionan que la principal razón por la cual no realizan actividad física es la pereza $(51 \%)$. Además, en una Encuesta Nacional de Salud (ENS) se indicó que, en España, el 40\% de la población adulta se declara sedentaria, ya que en su tiempo libre se dedican a ver televisión, ir al cine, entre otros; de igual forma, en esta población, permanecen sentados $(37,8 \%)$ en su mayoría. Biswas et al. (2015) menciona que "el tiempo de sedentarismo prolongado es perjudicial para la salud". Además, para Carson et al. (2016) "diferentes comportamientos sedentarios pueden tener impactos en varios indicadores de la salud". Asimismo, es necesario saber los beneficios que nos otorga la actividad física para la salud, así lo menciona Reynaga et al (2016).

Con respecto a la relación existente entre actividad física y deporte con el nivel de glucosa, se encontró una relación significativa, como se muestra en la tabla 5. Es coherente al estudio de Leiva et al, (2017), demostrando que existe una relación alta entre sedentarismo y glucosa. El no cumplimiento de actividad física se asocia a una mayor probabilidad de personas con sobrepeso y obesidad, y en un futuro personas diabéticas, con sedentarismo y falta de actividad física serán más propensas a desarrollar enfermedades crónicas no transmisibles. Sin embargo, Díaz et al. (2018) mencionan "que a mayor nivel de actividad física se asocia a un perfil metabólico saludable y por ende, reducir desarrollar sobrepeso, obesidad, diabetes y síndrome metabólico". De igual manera, Hernández et al. (2010) mencionan que "el ejercicio físico es pilar fundamental; se recomienda realizar ejercicios aeróbicos en los diabéticos, esta práctica trae múltiples beneficios, destacando la mejoría en el control metabólico y puede ayudar a prevenir las enfermedades". Para ello, existen estudios como el de Lindström et al. (2006) de intervención de estilo de vida que tuvo como resultado un $43 \%$ de reducción en la pérdida de peso y mayor actividad física.

Así mismo, se encontró una relación significativa entre actividad física y deporte con el IMC. De igual manera, Leiva et al. (2017) mencionaron que "los sujetos con inactividad física presentaron un mayor IMC siendo en su estudio de alta correlación, estos resultados son muy relevantes, porque muestra sobre los efectos que tiene el sedentarismo, en donde el gasto energético es menor". Entonces no cumplir con las recomendaciones de actividad física se asocia con mayores niveles de IMC, como menciona Díaz et al. (2018) en su estudio. Además, Heliodoro et al. (1999) hacen mención que hay una relación entre IMC y la actividad física donde se menciona que la actividad física es importante en las personas, teniendo un impacto favorable en los indicadores de la salud y así tener una mejor salud.

Referente a recreación y manejo de tiempo libre en este estudio, se encontró que el 52,6\% de la población estudiada es "saludable y muy saludable". Estudios casi similares a los de Sobejano et al. (2009), donde se observa que la mitad de la población es activa en su tiempo libre con una respuesta de $73.4 \%$. Con respecto a recreación y manejo de tiempo libre, el $47.4 \%$ "poco saludable y no saludable"; valores semejantes a los reportados por Maestre et al. (2015), donde la prevalencia de inactividad física en el tiempo libre fue $53.9 \%$. En este estudio se ve una asociación entre la falta de actividad física y el tiempo libre; así también, lo menciona Gómez et al. (2020), donde se identificó que el $25.9 \%$ de su población estudiada son insuficientemente activos; además, se menciona que es importante educar la promoción de la salud y de hábitos de vida saludable en su tiempo libre. Por otro lado, Sobejano et al, (2009), también, resalta que tener el conocimiento de las ventajas de realizar actividad física en el tiempo libre está asociado con la prevención de diferentes enfermedades; asimismo Wu et al. (2016) hacen mención que el equilibrio de trabajo y la recreación están significativamente asociadas, lo cual se debe promover un estilo de vida más saludable que mantenga ese equilibrio para así poder reducir enfermedades.

Con respecto a la relación entre recreación y manejo de tiempo libre e IMC, según Vidarte et al. (2012), en su estudio mencionaron que, "los niveles de dependencia y asociaciones entre el nivel de sedentarismo y el factor de riesgo IMC, existe una relación directamente proporcional entre variables antropométricas, fisiológicas y socioculturales y la práctica de la actividad física que se convierten en factores determinantes de los niveles de sedentarismo". Así mismo, Calero et al. (2016) realizaron un estudio con el objetivo y el diseño e implementación de práctica de un grupo de actividades físico y recreativas para disminuir el IMC de la obesidad, y los resultados de este estudio fueron favorables ya que si hubo una disminución favorable del IMC. 
Con respecto a la relación de recreación y manejo del tiempo libre y glucosa, según Zaranza et al. (2010), afirmaron que, personas con diabetes pueden mejorar la reducción de la glucemia, realizando actividades físicas en su tiempo libre. Asimismo, Baquero et al. (2017) reportaron en su estudio que "más del $50 \%$ de los funcionarios presentan una enfermedad relacionada con el sedentarismo a causa de falta de actividades físico-recreativas". Del mismo modo, Vidarte et al. (2012) mencionaron que "el $72.7 \%$ de la población de edades entre 18 a 60 años, tiene un estilo de vida sedentario". Entonces de este modo, Baquero et al. (2017), demostraron la importancia de implementar actividades físico-recreativas para reducir el porcentaje de casos ligados a enfermedades relacionadas al sedentarismo.

Con referencia al consumo de alcohol, tabaco y otras drogas, el $94.2 \%$ presentó una condición entre "muy saludables y saludables", valores similares a los de Castro et al. (2017), donde el $92.2 \%$ del grupo estudiado no fumaron y el $50 \%$ no consumía bebidas alcohólicas. Así mismo, Fernández et al. (2016), encontraron que en el $76.9 \%$ del grupo estudiado, no se evidenció el consumo de tabaco. También, esta investigación muestra que $5.7 \%$ de la población es "poco saludable y no saludable"; de igual forma, Castro et al. (2017) mencionan en su estudio que el 7.2 $\%$ tienen una dependencia al tabaco; además, no se encontró una relación con el estilo de vida saludable; sin embargo, Gil et al. (2019), reporta que el consumo de alcohol está asociado a la inactividad física de forma habitual. Asimismo, Lorenzini et al. (2015) en su investigación resaltaron que el tabaquismo tuvo una influencia importante (IMC). Los alumnos que no fumaban tuvieron un IMC mejor, comparado con aquellos que si fumaban. También en el estudio de Ruiz et al. (2018) reportaron que el consumo de alcohol está asociado al sobrepeso y obesidad. Con todo lo demostrado, Gil et al. (2019) evidenciaron la necesidad de promover programas de estilo de vida saludable que fomenten hábitos saludables.

Respecto al nivel de glucosa en este estudio se muestra que el $57.7 \%$ tiene el nivel de glucosa normal, de la misma forma Heredia (2016), reportaron un nivel alto de glucosa $(82.5 \%)$. Referente a los resultados de "prediabetes" se encontró que el $27.4 \%$ padecía de esta condición; de manera casi similar Sánchez et al. (2019) presentaron un 15\% con intolerancia a la glucosa (prediabetes), representando un alto riesgo de desarrollar diabetes en los siguientes años. También García et al. (2012), determinaron la alteración de glucosa en ayunas en una población urbana presentando un $29,2 \%$ de intolerancia a la glucosa, resultados muy semejantes a los reportados en esta investigación. Del mismo modo, Stepenka et al. (2018) demostraron que la prevalencia de prediabetes representó un $58.5 \%$ en la población evaluada. Además, los resultados presentados por Salinero et al. (2016), demostraron que niveles elevados de glucosa en sangre o prediabetes, representan una probabilidad alta de desarrollar diabetes en los siguientes 10 años. Por lo reportado, Stepenka et al. (2018), motivan a realizar intervención programas de prevención para evitar su avance. En cuanto a la diabetes, este estudio muestra que el $14.9 \%$ de los participantes la padece, de igual forma, Basto et al. (2016) encontraron valores aproximados en la prevalencia de diabetes $(13.7 \%)$, de los cuales el $30 \%$ desconocía de su enfermedad, así pues, se sugiere buscar una estrategia de diagnóstico oportuno, para la prevención de esta enfermedad. Para ello, se encontró estudios como Zaranza et al. (2010), que hay una reducción significativa en el nivel de glucosa en pacientes diabéticos.

Acerca del IMC, el $42.3 \%$ presenta "sobrepeso", hallazgos análogos a los presentados fueron encontrados por Goday et al. (2013) en los que la prevalencia de sobrepeso se encontró una prevalencia de obesidad en un $38.4 \%$. De igual manera, en un estudio llevado a cabo por Argüello et al. (2017), se evidenció que el $50.7 \%$ presentó sobrepeso; asimismo, en la investigación de Herrera et al. (2020) el 38,6 \% presentaba sobrepeso en estos dos estudios se identificó comportamientos negativos asociados a sus hábitos alimentarios; por esta razón, Leiva et al. (2017), en su estudio, donde también presentó un $52.4 \%$ con sobrepeso, hace referencia que la causa es debido a altos niveles de sedentarismo. Por consiguiente, Goday et al. (2013) mencionaron que "es necesario promover programas de prevención temprano". También en esta investigación, se encontró que el $23.4 \%$ presentan "obesidad de grado I, II, III", casi similar a lo reportado en los estudios de Herrera et al. (2020), presentando niveles de obesidad del $39.2 \%$, evidenciando que uno de los factores de riesgo encontrado fue el sedentarismo.

Asimismo, se encontró que el $34.3 \%$ de los participantes, presenta un estado normal. En 
el estudio de Argüello et al. (2017), reporta que un $36.9 \%$ es diagnosticado con IMC normal, lo cual es de gran importancia para prevenir complicaciones metabólicas. En efecto, como ya se mencionó la alta prevalencia de sobrepeso está relacionado con los malos hábitos; así lo menciona Lorenzini et al. (2015), en su estudio, la población estudiada sin distinción de género, presentaron sobrepeso, obesidad clase 1 y obesidad clase 2 (48.87\%). Estos resultados se relacionaron a los malos hábitos alimentarios; así mismo, el IMC se relacionó con el hábito del consumo de desayuno; el IMC fue mejor en los alumnos que desayunaban, en comparación con los que no lo hacían.

Referente al sueño, según la tabla 2, la minoría tiene un sueño inadecuado, de acuerdo a Jaime, et al. (2018), una mala calidad de sueño, puede presentar estrés, sabiendo que el sueño es primordial para regenerar las células de nuestro organismo. Ahora bien, Spiegel et al. (2004) declaran que la restricción del sueño puede llevar a una disminución de leptina, y una elevación de la grelina, causando un aumento del hambre y apetito, esto provoca el aumento de consumo de alimentos con alto contenido de carbohidratos, llevando a tener sobrepeso. Del mismo modo, en la tabla 5 encontramos la correlación del sueño con el nivel de glucosa y IMC. Según Juárez et al. (2009), el sueño puede dañar la tolerancia a la glucosa, altera las concentraciones de leptina y grelina, teniendo mayor susceptibilidad a tener síndrome metabólico y desarrollar obesidad. Por otra parte, (Salazar, 2014) encontró una relación inversa entre horas de sueño, presión sistólica y diastólica, en glucemia e IMC. Siendo una correlación entre baja y alta, la prevalencia de la obesidad ha ido en aumento paralelamente a la privación parcial crónica del sueño, parece haber mecanismos relacionados en ambos fenómenos.

Así mismo, en la tabla 2 se muestra que los hábitos alimentarios son adecuados, siendo más de la mitad de la población, presentando una mínima cantidad que no tiene buenos hábitos alimentarios. Se evidencia, en la tabla 5, que no existe correlación entre hábitos alimentarios y niveles de glucosa. Según López et al. (2011) la diabetes en adultos es más común y eso se debe a los factores de sedentarismo, sobrepeso, hiperglucemia, tabaquismo y hábitos alimentarios inadecuados. Un estudio realizado por Moreno Cruz (2013) demostró la influencia de los hábitos alimentarios tiene que ver mucho con la actividad física y el sobrepeso, se demostró que ellos tienen malos hábitos, consumen más de su requerimiento, se halló personas con IMC mayor a 25, presentando colesterol e hiperglicemia, por el mayor consumo de azúcar.

De igual manera, en la tabla 2 , encontró que más de la mitad de la población no tiene un autocuidado y cuidado médico adecuado. Mientras tanto, Escobar et al. (2013) demostraron que las mujeres realizan y tienen más autocuidado, pero no acuden al médico porque se sienten bien de salud, son ellas las que practican el autocuidado y muy pocas veces lo hacen los hombres. Por su parte, Villalobos et al. (2019) han reportado que muchos no tiene el autocuidado por los siguientes factores; la baja escolaridad, bajo estado económico y lengua indígena, estos factores incrementan no realizar acciones de autocuidado, por ello se debe implementar programas de educación y mejora de atención por parte del personal de salud. Así mismo, la tabla 5 , demuestra una correlación entre el autocuidado y cuidado médico y nivel de glucosa, según soler (2016) es posible que un conocimiento bajo de la diabetes representa ausencia de autocuidado, por cuanto, la educación y la promoción de la salud es primordial, debido a que se relaciona con acciones en beneficio de la salud, tales como actividad física, nutrición y el autocontrol.

Por otro lado, se encontró correlación entre el autocuidado y cuidado médico con respecto al IMC. Según Macías et al. (2018), se sabe que el sobrepeso es el principal problema que afecta a la salud de toda la población y tiene una relación con el manejo de autocuidado, se observa que la mayoría de la población estudiada presenta sobrepeso, esto debido a sus hábitos alimentarios y un déficit en el nivel de cuidado.

Esta investigación demostró que el estilo de vida está relacionado a los niveles de glucosa y el IMC de los participantes. Además, el estudio demuestra que la falta de actividad física está relacionado al aumento de IMC que a la vez se asocia a los niveles de glucosa elevados. Por lo tanto, si se hace una modificación del estilo de vida, condición física y deporte, recreación y manejo del tiempo libre, consumo de alcohol y otras drogas, sueño, hábitos alimentarios y autocuidado y cuidado médico, puede ser de gran ayuda para la prevención de enfermedades metabólicas. 


\section{Declaración de financiamiento y de conflictos de interés:}

El estudio fue financiado por los autores, quienes declaran no tener conflictos de interés

\section{Correspondencia:}

Carla Hurtado Casanca

Correo electrónico:

arellyhurtado@upeu.edu.pe

Noelene Atoc Ventocilla

Correo electrónico:

noeatoc@gmail.com

Mery Rodríguez Vásquez

Correo electrónico:

meryrv@upeu.edu.pe

\section{REFERENCIAS BIBLIOGRÁFICAS}

Organización Mundial de la Salud (2020) Obesidad y sobrepeso Datos y cifras. Organ Mundial de la Salud. ;(Imc):1-7. recuperado de: https://www. who.int/es/news-room/fact-sheets/detail/obesityand-overweight

Ccorahua M, Atamari N, Miranda I, Campero A.(2019) Prevalencia de la diabetes mellitus tipo 2 en población menor de 30 años para el período de 2005 a 2018 con datos del Ministerio de Salud de Perú. Medwave. 2019;19(10):e7723. Recuperado de: https://www.medwave.cl/link.cgi/Medwave/ Estudios/Investigacion/7723.act

Moreno M.(2012) Definición y clasificación de la obesidad. Rev Médica Clínica Las Condes.Mar;23(2):124-8.Recuperado de: https://reader.elsevier.com/reader/sd/pii/ S0716864012702882?token=0FE0E634564ABE B3116C717B8E32121344DFF4446756EC5B831 77DB61DD97B23D78C254EA5599DBE2787A47 2B839162A

Durán S, Carrasco E, Araya M. (2012) Alimentación y diabetes. Nutr Hosp. 27(4):1031-6. Recuperado de:http://scielo.isciii.es/pdf/nh/v27n4/10_ revision09.pdf

Reynaga P, Arévalo E, Verdesoto A, Jiménez I, Preciado M, Morales J.(2016) Beneficios psicológicos de la actividad física en el trabajo de un centro educativo. Retos. 2016;2041(30):2036.Recuperado de: file:///C:/Users/USER/ Downloads/Dialnet-BeneficiosPsicologicosDeLa ActividadFisicaEnElTraba-5529626.pdf

Elizondo J, Guillén F, Aguinaga I.(2005) Prevalencia de actividad física y su relación con variables sociodemográficas y estilos de vida en la población de 18 a 65 años de Pamplona. Rev Esp Salud Pública. 2005;79(5):559-67. Recuperado de: http://scielo.isciii.es/pdf/resp/v79n5/original4. pdf

Aguirre M, Crespo P. (2015) Prevalencia de síndrome metabólico en adultos. (tesis de grado, universidad de paraguay) Recuperado de: http:// dspace.uazuay.edu.ec/handle/datos/4217

Triviño L, Dosman V, Uribe Y, Agredo R, Jerez A, Ramírez R. Estudio del estilo de vida y su relación con factores de riesgo de síndrome metabólico en adultos de mediana edad. ACTA MÉDICA Colomb. 2009;34(4):1-6. Recuperado de: http:// www.scielo.org.co/pdf/amc/v34n4/v34n4a2.pdf

Silva J, Ayala M, Mendonça J, Partezani R. Estilo de vida asociado a autoestima y variables demográficas en estudiantes de enfermería. Rev Científica Ciencias la Salud. 2017;9(2):72-8. Recuperado de: https://revistas.upeu.edu.pe/ index.php/rc_salud/article/view/233

Ribeiro, G; Rota L. (2019)Lifestyle and nutritional status of employees of a chain of banks in Pelotas, Rio Grande do Sul, Brazil. Rev Bras Med do Trab. 2019;17(1):45-53.Recuperado de: https://www. ncbi.nlm.nih.gov/pmc/articles/PMC7138499/

Barragán L, González M, Estrada S, Hernández Y, Hernández E, Ríos J, Flores M.(2015) Estilo de vida y dimensiones, en estudiantes universitarios de área de la salud. Ciencia y Humanismo en la Salud. 2015;2(2):53-63. Recuperado de:

Mora R.(2012) Medicina del estilo de vida: la importancia de considerar todas las causas de la enfermedad. Rev Psiquiatr Salud Ment. 2012;5(1):48-52. Recuperado de: http://dx.doi. org/10.1016/j.rpsm.2011.04.002

Reséndiz E, Aguilera P, Rocher M. Estilo de vida e índice de masa corporal de una población de adultos del sur de Tamaulipas, México - Dialnet. Aquichan [Internet]. 2010 [cited 2020 Feb 10];10(3):244-52. Recuperado de: https://dialnet. unirioja.es/servlet/articulo?codigo $=3400996$

Ramírez M, Carvajalino M, Coronado M, Gómez C, Mayorga L, Medina R. (2011) Estilo de vida actual de los pacientes con diabetes mellitus tipo 2. Rev Cienc y Cuid ;8(1):21-8. Recuperado de: https:// revistas.ufps.edu.co/index.php/cienciaycuidado/ article/view/466

Molina J, Sánchez S, Herreros M, Vizcarro D, López C.(2016) Prevalencia de actividad física en profesionales de atención primaria de Cataluña. Semergen . ;43(5):352-7. Recuperado de: http:// dx.doi.org/10.1016/j.semerg.2016.04.026 
Velandia M, Arenas J, Ortega N.(2015) Estilos de vida en los estudiantes de enfermería. Rev Cienc y Cuid.;12(1):27. Recuperado de: https://revistas. ufps.edu.co/index.php/cienciaycuidado/article/ view $/ 320$

Biswas A, Oh P, Faulkner G, Bajaj R, Silver M, Mitchell $M$, et al. Sedentary time and its association with risk for disease incidence, mortality, and hospitalization in adults. Ann Intern Med. 2015;162(2):123-32. Recuperado de: https://pubmed.ncbi.nlm.nih. gov/25599350/

Carson V, Hunter S, Kuzik N, Gray C, Poitras V, Chaput J, Saunders T. , Latzmarzyk P.,Okely A., Connor S., Kho M.,Sampson M., Lee H.,Tremblay M (2016) Systematic review of sedentary behaviour and health indicators in school-aged children and youth: An update. Appl Physiol Nutr Metab. ;41(6):S240 65. Recuperado de: https://pubmed.ncbi.nlm.nih. gov/27306432/

Leiva A, Martínez M, Cristi C, Salas C, Ramírez R, Martínez X, Aguilar N., Celis C.,(2017) El sedentarismo se asocia a un incremento de factores de riesgo cardiovascular y metabólicos independiente de los niveles de actividad física. Rev Med Chil. 145(4):458-67. Recuperado de: https://scielo.conicyt.cl/scielo.php?script=sci_ arttext\&pid=S0034-98872017000400006

Díaz X, Petermann F, Leiva A, Garrido A, Salas C, Martínez M, et al.(2018) No cumplir con las recomendaciones de actividad física se asocia a mayores niveles de obesidad, diabetes, hipertensión y síndrome metabólico en población chilena. Rev Med Chil.146(5):585-95. Recuperado de: https://scielo.conicyt.cl/scielo.php?script=sci_ arttext\&pid=S0034-98872018000500585

Lindström J, llanne P, Peltonen M, Aunola S, Eriksson $\mathrm{J}$, Hemiö K, et al.(2006) Sustained reduction in the incidence of type 2 diabetes by lifestyle intervention: follow-up of the Finnish Diabetes Prevention Study. Lancet. 368(9548):1673-9.Recuperado de: https:// pubmed.ncbi.nlm.nih.gov/17098085/

Heliodoro M, Esparza J, ValenciaM.(1999)Antropometría y composición corporal en personas mayores de 60 años. Importancia de la actividad física. Salud Publica Mex. 41(4):309-16. Recuperado de: https:// scielosp.org/pdf/spm/1999.v41n4/309-316/es

Sobejano I, Moreno C, Viñes J, Grijalba A, Amézqueta C, Serrano M. (2009)Estudio poblacional de actividad física en tiempo libre. Gac Sanit. 23(2):127-32. Recuperado de: https://analytics. scielo.org/?journal=0213-9111\&collection=esp

Maestre C, Regidor E, Cuthill F. (2015) Desigualdad En La Prevalencia De Sedentarismo Durante EI Tiempo Libre En Población Adulta Española Según Su Nivel De Educación. Diferencias Entre 2002 Y 2012. Rev Esp Salud Pública.2012:259-69. Recuperado de: http://scielo.isciii.es/scielo.php?script=sci_
abstract\&pid=S1135-57272015000300004

Gómez M, Sánchez D, Labisa A. (2020) Actividad física en tiempo libre en estudiantes universitarios colombianos. Retos nuevas tendencias en Educ física, Deport y recreación 2041(37):181-9. Recuperado de: https://dialnet.unirioja.es/descarga/ articulo/7243266.pdf\%0Ahttps://dialnet.unirioja.es/ servlet/extart?codigo $=7243266$

Vidarte J, Vélez C, Parra J. (2012) Niveles de sedentarismo en población de 18 a 60 años. Rev. Salud Pública. Recuperado de: https://www. scielosp.org/pdf/rsap/2012.v14n3/417-428

Calero S, Maldonado I, Fernández A, Rodríguez Á, Otáñez N (2016). Actividades físico-recreativas para disminuir la obesidad en mujeres entre los 35-50 años de edad. Rev Cuba Investig Biomédicas. 35(4):375-86. Recuperado de: http://scielo.sld.cu/scielo.php?script=sci_ arttext\&pid=S0864-03002016000400008

Zaranza L, Vaz C, Foss M, Zanetti M, Foss C.(2010) Reducción de la Presión Arterial, del IMC y de la Glucosa tras Entrenamiento Aeróbico en Mujeres Adultas Mayores con Diabetes Tipo 2. Arq Bras Cardiol. 95(5):563-70. Recuperado de: http://www. arquivosonline.com.br/espanol/2010/9505/pdf/ e9505002.pdf

Baquero F, Chávez E. (2017) Nivel De Sedentarismo Y Propuesta De Intervención Físico- Recreativa En Servidores Públicos De Santa Rosa. Rev Cuba Investig Biomédicas 36(2):1-14. Recuperado de: http://scielo.sld.cu/pdf/ibi/v36n2/ibi09217.pdf

Castro M, Puertas P, Ubago J, Pérez A, Linares M, Zurita F. (2017) Consumo de tabaco y alcohol en universitarios. J Sport Heal Res.9(1):15162. Recuperado de: https://www.researchgate. net/publication/317512079_CONSUMO_DE TABACO_Y_ALCOHOL_EN_ÜNIVERSITARIOS_ CONSUMPTION_OF TOBACCO_AND ALCOHOL_IN_COLLEGES

Fernández E, Molerio O, Fabelo J, Sanchez D (2016). Consumo de tabaco y alcohol en estudiantes universitarios cubanos. Rev del Hosp Psiquiatr la Habana .13(2):1-11. Recuperado de: https://www. medigraphic.com/pdfs/revhospsihab/hph-2016/ hph162a.pdf

Gil P, Prieto A, Dos Santos S, Serra J, Aguilar M, Díaz A. (2019) Hábitos y comportamientos relacionados con la salud de los adolescentes en su tiempo de ocio. An Psicol 35(1):140-7. Recuperado de: https://revistas.um.es/analesps/article/view/301611

Lorenzini R, Betancur D, Chel L, Segura M, Castellanos A. (2015) Estado nutricional en relación con el estilo de vida de estudiantes universitarios mexicanos. Nutr Hosp. 32(1):94-100. Recuperado de: $\quad$ http://scielo.isciii.es/scielo.php?script=sci arttext\&pid=S0212-16112015000700015 
Ruiz M, Mora G, Gómez D. (2018) Asociación del consumo de alcohol y tabaco con la obesidad en adultos de Cartagena de Indias, Colombia. Salud Uninorte.34(1):100-11. Recuperado de: http:// www.scielo.org.co/pdf/sun/v34n1/2011-7531sun-34-01-109.pdf

Heredia S, Robalino M, Hidalgo M, Proaño F, Antamba E, Yánez P (2016). Caracterización Del Perfil Lipídico, Índice De Corporal Y Nivel De Glucosa En Afiliados Del Instituto Ecuatoriano De Seguridad Social ( less ) Riobamba, 2014 Qualitas vol.12:124134. Recuperado de: https://www.researchgate. net/publication/313842976_Caracterizacion_ del_perfil_lipidico_indice_de_masa_corporal_y_ nivel_de_glucosa_en_afiliados_dēl_Instituto_ Ecuatoriano_de_Seguridad_Social_IESS_Riobamba_2014_como_parametros_indicadores_ de_su_estado_de

Sánchez B, Chico G, RodríguezA, Sámano R, Veruete D, Morales R. (2019) Detección de riesgo de diabetes tipo 2 y su relación con alteraciones metabólicas en enfermeras. Rev Lat Am Enfermagem. 27. Recuperado de: https://www.scielo.br/pdf/rlae/v27/ es_1518-8345-rlae-27-e3161.pdf

García H, Christelle N, Tamborero G, Hirales O, Salinas J, Soto E. (2012) Frequency of diabetes, impaired fasting glucose, and glucose intolerance in high-risk groups identified by a FINDRISC survey in Puebla City, Mexico. Diabetes, Metab Syndr Obes Targets Ther. 2012;5:403-6. Recuperado de: https:// pubmed.ncbi.nlm.nih.gov/23204848/

Stepenka V, Rivas Y, Zapata Y, Añez L, Casal J, Sindas M, et al. (2018) Prevalencia de Prediabetes y Diabetes Mellitus en la región de Zulia, Venezuela . Medicina interna 34(3):179-83. Recuperado de: https://www.svmi.web.ve/ojs/index.php/medint/ article/view/483

Salinero M, Burgos C, Lahoz C, Mostaza J, Abánades C, Laguna F, et al (2016). Performance of the finnish diabetes risk score and a simplified finnish diabetes risk score in a community-based, cross-sectional programme for screening of undiagnosed type 2 diabetes mellitus and dysglycaemia in madrid, Spain: The SPREDIA-2 study. PLoS One. 11(7):117. Recuperado de: https://journals.plos.org/ plosone/article?id=10.1371/journal. pone. 0158489

Basto A, Barrientos T, Rojas R, Aguilar C, López N, De la Cruz V, et al. Prevalencia de diabetes y descontrol glucemico en México: Resultados de la Ensanut 2016. Salud Publica Mex. 2020;62(1):50-9. Recuperado de: https://www.medigraphic.com/cgibin/new/resumen.cgi?IDARTICULO=91020

Herrera B, Ruíz S, Zapién A, Sánchez G, Bernardino $H$. (2020) Factores de riesgo para obesidad en población femenina del Istmo de Tehuantepec, Oaxaca, México. Acta Med Costarric. 2020;62:13-
7. Recuperado de: https://www.scielo.sa.cr/pdf/ amc/v62n1/0001-6002-amc-62-01-13.pdf

Jaime L, Juárez D, Téllez A. (2018) influencia del estrés y el optimismo sobre la calidad de sueño de mujeres con cáncer de mama. Psicooncologia. 15(2):249-60.Recuperado de: file:///C:/Users/ USER/Downloads/61434-Texto $\% 20 \mathrm{del} \% 20$ art\%C3\%ADculo-4564456555041-2-10-201810 16.pdf

Spiegel K, Tasali E, Penev P, Van Cauter E. (2004) Brief communication: Sleep curtailment in healthy young men is associated with decreased leptin levels, elevated ghrelin levels, and increased hunger and appetite. Ann Intern Med. 141(11):846-50. Recuperado de: https://pubmed.ncbi.nlm.nih. gov/15583226/

Juárez E, Jiménez N, Lozano J, Fernández M. (2009) Relación entre horas de sueño y síndrome metabólico. Med Interna Mex. 25(1):9-16. Recuperado de: https://www.medigraphic.com/cgibin/new/resumen.cgi?IDARTICULO=25136

López H, Pérez R, Monroy R. (2011) Factores De Riesgo Y Hábitos Alimentarios En Personas De 25 A 35 Años, Con Y Sin Antecedentes De Diabetes Mellitus Tipo 2., 12(2). Rev salud Publica Nutr. Recuperado de: https://www.medigraphic.com/cgibin/new/resumen.cgi?IDARTICULO=30347

Escobar M, Pico M. (2013) Autocuidado de la salud en jóvenes universitarios, Manizales, 2010-2011. Self-care Univ students Manizales, 2010-2011 31(2):178-86. Rev. Fac Nac Salud Pública. Recuperado de: http://search.ebscohost.com/login. aspx?direct=true \&db=a9h\&AN=92621803\&lang=e s\&site=ehost-live

Soler Y, Pérez E, Lopez M, Quezada D. (2016) Conocimientos y autocuidado en pacientes con diabetes mellitus tipo 2. Arch Médico Camagüey. 20(3):244-52. Recuperado de: http://scielo.sld.cu/scielo.php?script=sci_ arttext\&pid=S1025-02552016000300004

Macías A, Rincón T, Sanipatin J, Zambrano R. (2018) Sobrepeso y autocuidado en estudiantes de la carrera enfermería. Rev Vive. 1(3):139-46. Recuperado de: https://revistavive.org/index.php/ revistavive/article/view/14

Recibido: 10/06/2020

Aceptado: 20/09/2020 